\title{
Selective Study of Fe Atoms at the Interfaces of an Fe/Ir(100) Superlattice by means of Diffraction Anomalous Fine Structure
}

\author{
H. Renevier, ${ }^{1,2, *}$ J. L. Hodeau, ${ }^{1,3}$ P. Wolfers, ${ }^{1}$ S. Andrieu, ${ }^{4}$ J. Weigelt, ${ }^{5}$ and R. Frahm ${ }^{5}$ \\ ${ }^{1}$ Laboratoire de Cristallographie, Centre National de la Recherche Scientifique, B.P. 166, 38042 Grenoble Cedex 09, France \\ ${ }^{2}$ Université Joseph Fourier, B.P. 53, 38041 Grenoble Cedex 09, France \\ ${ }^{3}$ European Synchrotron Radiation Facility, B.P. 220, 380423 Grenoble Cedex, France \\ ${ }^{4}$ LMPSM, URA 155, Université de Nancy I, B.P. 239, 54506 Vandoeuvre, Nancy Cedex, France \\ ${ }^{5}$ Hamburger Synchrotron Strahlungslabor HASYLAB am DESY, Notkestrasse 85, 22603 Hamburg, Germany
}

(Received 26 August 1996)

\begin{abstract}
Studies of superlattices are important in order to synthesize 3D metals in a new crystalline structure which may exhibit exotic magnetic properties. The challenge is to relate these properties to the details of the chemical gradient and the local strain. We report the use of diffraction anomalous fine structure (DAFS) spectroscopy and a crystallographic analysis of the DAFS data to separate the x-ray-absorption fine structure (XAFS)-like information about Fe atoms located at different crystallographic sites of an $\mathrm{Fe} / \mathrm{Ir}(100)$ superlattice. We obtained the first and the second (in the plane of growth) nearest-neighbor distances of $\mathrm{Fe}$ atoms at the Ir-Fe interface. [S0031-9007(97)02789-0]
\end{abstract}

PACS numbers: 61.10.-i, 07.85.Jy, 78.70.Dm

Diffraction anomalous fine structure (DAFS) spectroscopy provides a site-selective $\mathrm{x}$-ray-absorption fine structure (XAFS)-like information about the local atomic environment and the chemical state of the "edge atom" through resonant x-ray scattering and diffraction. Several years after a few pioneering works [1], Stragier et al. [2] presented an elegant demonstration of this method on a copper single crystal and later, several groups applied it on different types of samples like thin films, superlattices [3,4], powders [5], and single crystals [6]. Most results were obtained with materials of which structural information could be extracted with single site-selective Bragg reflections. Site-selective information with real samples was successfully extracted in the DANES (diffraction near edge structure) region of the spectrum; however, due to insufficient statistics no information in the EDAFS (extended diffraction anomalous fine structure) region could be obtained [6]. Regarding superlattices, only the spatial selectivity of the DAFS has been used. The first DAFS experiments on several pseudomorphic Fe/Ir(100) superlattices were carried out at the Ir edge to determine separately the Ir crystallographic parameters in the buffer and the superlattice [4] (spatial selectivity). We present here a crystallographic-based site-selective EDAFS study of an $\mathrm{Fe} / \operatorname{Ir}(100)$ superlattice to separate the XAFS-like information about $\mathrm{Fe}$ at the Ir-Fe interfaces.

$\mathrm{Fe} / \mathrm{Ir}$ superlattices were synthesized to stabilize $\mathrm{Fe}$ in new crystalline structures with the aim of obtaining new magnetic properties. Different strained Fe/Ir structures may be synthesized by varying the thicknesses of the Fe and Ir layers [7]. It has been shown that Fe relaxes into a bcc structure (as bulk Fe) in thick Fe layers (more than five atomic planes) grown at most on three Ir atomic planes, whereas in thin Fe layers (less than five atomic planes) Fe has a body centered tetragonal structure (bct) with a $c / a$ ratio near 1.25 [8] (pseudomorphic regime).
For intermediate thicknesses with thick Fe and Ir layers (more than five atomic planes each) the $\mathrm{Fe}$ structure is still not accurately known. High resolution electron microscopy experiments showed that the superlattices are damaged with the disappearance of $2 \mathrm{D}$ growth and the presence of a large amount of dislocations [7]. Regarding magnetic properties, a transition from a nonmagnetic state at room temperature to a low-spin ferromagnetic state was observed in the strained bct phase. A larger Fe magnetic moment exists in the bcc phase, and it has been shown that the magnetization is correlated to the Ir thickness. However, the relation between magnetism and structure is still not well understood. Furthermore, results of Mössbauer experiments indicated that several magnetic sites are present in the samples.

We carried out a DAFS study of an $\mathrm{Fe} / \operatorname{Ir}(100)$ superlattice with $\mathrm{Fe}$ in the bcc phase for detecting changes of the Fe local structure inside the Fe layers. For that purpose we recorded a set of DAFS spectrum at the Fe $K$ edge with several reflections. The composition gradient, the bulk absorbance of the sample, and the contribution of Fe sites to the measured reflections were obtained by refining simultaneously three diffraction data together with the smooth parts of five DAFS spectra. We show that DAFS measurements give precise site-selective information which can be used for the determination of the local strain variations inside a superlattice.

The Fe/Ir superlattice studied was grown by molecular beam epitaxy on an $\mathrm{MgO}(100)$ substrate (without any Ir buffer) and made of $80 \mathrm{Fe} / \mathrm{Ir}$ bilayers with a total thickness of $0.24 \mu \mathrm{m}$. The diffraction spectra show (see the inset of Fig. 1 as an example) several well defined superlattice $(00 l)$ reflections indicating a good epitaxy. Considering the nominal $\mathrm{Fe}$ and Ir layer thicknesses $(\approx 28$ and $\approx 3 \AA$, respectively), the Fe structure is expected to be bcc in average. 


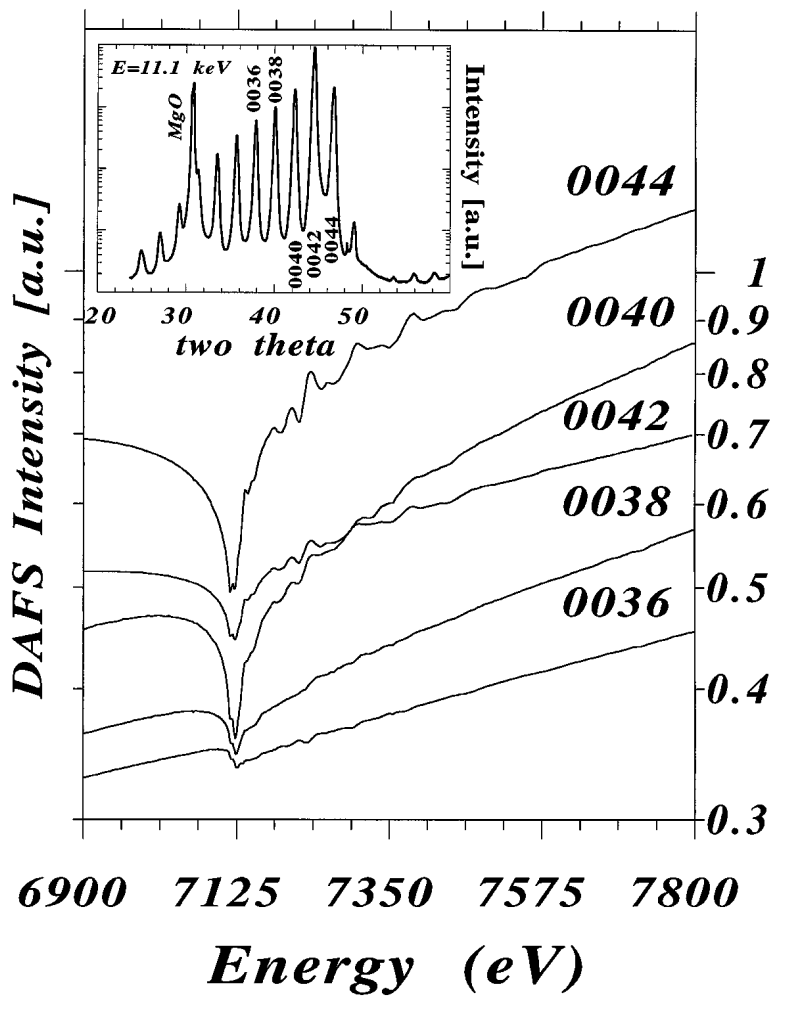

FIG. 1. Raw DAFS spectra measured at the Fe $K$-edge with the reflections 0036 to 0044 of the $\mathrm{Fe} / \mathrm{Ir}$ superlattice (topDAFS-scan procedure, logarithmic scale). For presentation, intensities of the DAFS spectra were rescaled. A part of the diffraction spectrum at $11.1 \mathrm{keV}$ is also shown (inset).

The DAFS spectra were recorded at the x-ray undulator beam line BW1 at HASYLAB [9]. The intensities were recorded in reflection mode, with the polarization perpendicular to the plane of diffraction. A complete diffraction spectra (from the reflections 0012 to 00160) was collected at an energy of $11000 \mathrm{eV}$ close to the Ir $L_{\mathrm{III}}$-edge $(11212 \mathrm{eV})$ at the beam line D2AM at the ESRF [10], and two diffraction spectra of reflections close to the first order intereticular distance (0034 to 0044) were collected at HASYLAB at two energies of 6900 and $7300 \mathrm{eV}$ above and below the Fe $K$-edge $(7112 \mathrm{eV})$. Since the shape of the superlattice reflections exhibited a large FWHM $\left(\approx 0.5^{\circ}\right)$, we used the diffracted intensity at the maximum of the reflections while scanning the beam energy (top-DAFS-scan procedure). DAFS spectra of five reflections (from 0034 to 0044$)$ were collected at the $\mathrm{Fe} K$-edge with a varying step width (down to $2 \mathrm{eV}$ ) in the $6900-7800 \mathrm{eV}$ range. Because of the mosaicity, inhomogenous absorption, or too small reflections widths, the top-DAFS-scan procedure cannot be applied with all samples [6], but is well suited for superlattices, monolayers, or thin films. The fluorescence signal was simultaneously recorded for energy calibration and the absorption correction.

Changes in the diffracted intensities near an absorption edge are related to those of all anomalous atomic scattering factors. In the forward scattering limit, the atomic scattering factor of an atom $A$ on site $j$ may be split above the edge in smooth and oscillatory parts $[1(\mathrm{~b}), 2,3]$ :

$$
\begin{aligned}
f_{A j}(\mathbf{Q}, E)= & f_{0 A}(\mathbf{Q})+f_{0 A}^{\prime}(E)+i f_{0 A}^{\prime \prime}(E) \\
& +\Delta f_{0 A}^{\prime \prime}(E)\left[\chi_{A_{j}}^{\prime}(E)+i \chi_{A_{j}}^{\prime \prime}(E)\right],
\end{aligned}
$$

where $f_{0 A}$ is the Thomson scattering, $f_{0 A}^{\prime}$ and $f_{0 A}^{\prime \prime}$ are the "bare" atom anomalous corrections to $f_{A_{j}}$, and $\Delta f_{0 A}^{\prime \prime}$ is the contribution of the resonant electronic transition alone. $\mathbf{Q}$ is the scattering vector and $E$ the energy of the incident beam; $\chi_{A_{j}}^{\prime}+i \chi_{A_{j}}^{\prime \prime}$ is the complex fine structure, $\chi_{A_{j}}^{\prime \prime}$ is equivalent to the extended x-ray-absorption fine structure (EXAFS) signal and $\chi_{A_{j}}^{\prime}$ is related to $\chi_{A_{j}}^{\prime \prime}$ via the Kramers-Kronig transform. From (1) the structure factor $F(\mathbf{Q}, E)$ is divided into smooth and oscillatory parts: $F(\mathbf{Q}, E)=F_{0}(\mathbf{Q}, E)+$ $\alpha(\mathbf{Q}) \Delta f_{0 A}^{\prime \prime}(E)\left[\chi^{\prime}(\mathbf{Q}, E)+i \chi^{\prime \prime}(\mathbf{Q}, E)\right], \quad \chi^{\prime}+i \chi^{\prime \prime}=$ $\frac{1}{\alpha} \sum_{j} \alpha_{A_{j}}\left[\chi_{A_{j}}^{\prime}+i \chi_{A_{j}}^{\prime \prime}\right]$ and $\alpha=\sum_{j} \alpha_{A_{j}}$. The summation runs over all anomalous atoms $j$ in the cell. When considering a centrosymmetric structure, $\alpha_{A_{j}}(\mathbf{Q})=c_{A_{j}} N_{A_{j}} \cos \left(\mathbf{Q} \cdot \mathbf{r}_{A_{j}}\right) \exp \left(-M_{A_{j}} Q^{2}\right)$, where $c_{A_{j}}$ is the occupation factor of atom $A$ on site $j, N_{A_{j}}$ is a multiplicity number, and $\exp \left(-M_{A_{j}} Q^{2}\right)$ is the crystallographic Debye-Waller factor. The measured intensity is proportional to the square modulus of the structure factor $\left(|F|^{2}\right)$ times several correction terms:

$$
I(\mathbf{Q}, E)=S(E) D(E) A(\mathbf{Q}, E) L P(\mathbf{Q}) \lambda^{3}|F(\mathbf{Q}, E)|^{2},
$$

where $S$ is a scale factor, $D$ and $A$ are the corrections for the detector efficiency and the bulk absorbance of the sample together with the geometrical effects, respectively, and $L P$ is the Lorentz-polarization correction. The crystallographic structure was obtained by fitting the calculated intensities, using Eq. (2) in which $F$ has been replaced by $F_{0}$, to the Bragg integrated intensities of the diffraction data and to the DAFS spectra. All anomalous corrections $f_{0}^{\prime}(E)$ and $f_{0}^{\prime \prime}(E)$ for calculating $F_{0}$ were the theoretical values calculated by Cromer's FPRIME program [11] (convolved with a Lorentzian function). The EDAFS oscillations $\left[\chi_{\mathbf{Q}}(E)\right]$ were extracted and normalized according to the following formula for a centrosymmetric structure:

$$
\chi_{\mathbf{Q}}(E)=\chi^{\prime}+\varepsilon_{\mathbf{Q}} \chi^{\prime \prime}=\left(\frac{I_{a c}-I_{a c 0}}{I_{a c 0}}\right) \frac{\left|F_{0}\right|^{2}}{2 \alpha \operatorname{Re}\left(F_{0}\right) \Delta f_{0 A}^{\prime \prime}},
$$

where $\varepsilon_{\mathbf{Q}}(E)=\operatorname{Im}\left(F_{0}\right) / \operatorname{Re}\left(F_{0}\right), I_{a c}$ is the measured intensity corrected for absorption, and $I_{a c 0}$ is the smooth part of $I_{a c}$. In the above treatment leading to Eq. (3), $|F|^{2}$ is expanded and the terms of order $\chi^{12}$ and $\chi^{\prime \prime 2}$ are neglected.

The next step is to solve for $\chi_{A_{j}}^{\prime}$ and $\chi_{A_{j}}^{\prime \prime}$ a linear system of $M$ equations of the form of Eq. (3) and $2 N$ parameters for each energy, where $M$ is the number of $\chi_{\mathbf{Q}}(E)$ EDAFS spectra and $N$ is the number of inequivalent anomalous site $j$. The Kramers-Kronig relation between $\chi_{A_{j}}^{\prime}$ and $\chi_{A_{j}}^{\prime \prime}$ is used as an additional constraint. 
The crystallographic structure has been refined to accurately correct for absorption the DAFS spectra (with the refined Ir and Fe occupation factors) and to determine the $\alpha_{A_{j}} / \alpha$ coefficients in order to separate the site dependent XAFS-like information. A centered tetragonal centrosymmetric cell with the space group $I 4 / \mathrm{m}$ has been used with the $c$ axis along the direction of growth. The final refinement gave a $1 / \sigma$-weight $R$-factor of $4.5 \%$. In this description the superlattice cell along the $c$ axis is made of two $\mathrm{Fe} / \mathrm{Ir}$ bilayers and only the $00 \mathrm{l}$ reflections with $l=2 n$ exist. The superlattice periodicity $c$ was found to be $2 \times 30.989 \AA$ and made of 34 atomic planes filled with $\mathrm{Fe}$ atoms plus eight containing at whole $30 \%$ of Ir atoms. Figure 2 shows one-half of the structure along the $c$ axis and the [110] direction of the body centered tetragonal structure. Results of the refinements show that for this sample which contains few Ir atoms, there is no sharp interfaces. The Ir rich planes (site 11) are filled with $42(3) \%$ of Ir atoms and site 10 with $17(4) \%$. In Table I the relative contribution $\left(\alpha_{\mathrm{Fe} 11} / \alpha\right)$ of $\mathrm{Fe}$ on site 11 is reported (the contribution of all other $\mathrm{Fe}$ sites is $\left.\alpha_{\mathrm{Fe} 1-10} / \alpha=1-\alpha_{\mathrm{Fe} 11} / \alpha\right)$. With the five available reflections only two sites have been determined, the reflection 0036 indicating a strong distortion of the Fe11 local atomic environment. As we could expect Fe layers mainly contribute to the Bragg peak which correspond to an interplanar distance close to the bcc Fe $d_{002}\left(d_{002}=\right.$ $1.44 \AA$ ), whereas the Ir rich planes contribute to the weak reflections 0036 and 0038 , which therefore contain the detail of the structure at the interfaces. This result points out the importance of using DAFS spectra for structural refinements: The smooth behavior of the DAFS spectra gives precise crystallographic information about anomalous sites contributing to the reflection, i.e., on the positions, the occupation, and the Debye-Waller factors. Including the 0038 and 0036 DAFS spectra (Fe $K$-edge) into the experimental diffraction data we have decreased the correlation between the occupation and the DebyeWaller of Fe at the interface (98\% to $85 \%$ ).

Raw DAFS spectra are shown in Fig. 1. Large DAFS oscillations can clearly be seen on reflections 0040 to 0044. The 0036 reflection still represents weak oscillations which do contains DAFS information, but are affected by the superlattice absorption. Figure 3 shows the five EDAFS oscillation spectra corrected for the fluores-

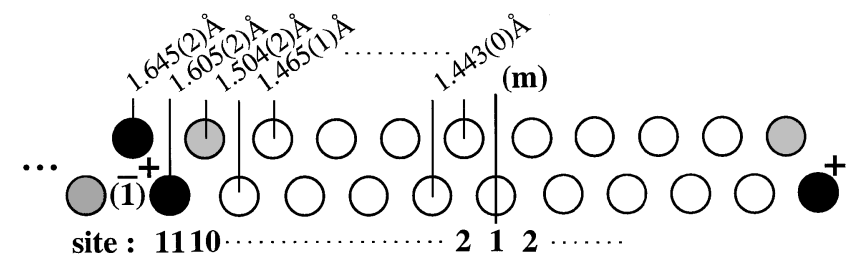

FIG. 2. Schematic view of one-half of the structure along the $c$ axis and the [110] direction of the bct structure. Sites 11 and 10 contain $42 \%$ and $17 \%$ Ir atoms, respectively.
TABLE I. The total phase of all anomalous Fe atoms $(\alpha)$ and the relative contributions of site $\mathrm{Fe} 11$ at the interfaces to the Bragg reflections $\left(\alpha_{\mathrm{Fe} 11} / \alpha\right)$.

\begin{tabular}{crrr}
\hline \hline $00 l$ & $\alpha$ & $\alpha_{\mathrm{Fe} 11} / \alpha$ & $d_{00 l}(\AA)$ \\
\hline 0036 & $-1.59(6)$ & $1.32(7)$ & 1.722 \\
0038 & $-4.06(6)$ & $0.50(2)$ & 1.631 \\
0040 & $-9.56(6)$ & $0.20(1)$ & 1.549 \\
0042 & $-28.84(2)$ & $0.06(0)$ & 1.476 \\
0044 & $21.36(1)$ & $-0.08(0)$ & 1.409 \\
\hline \hline
\end{tabular}

cence contaminated background and for the bulk absorption of the sample, and normalized according to Eq. (3). On going from the 0044 to the 0036 a continuous change of the spectra is visible showing the existence of different Fe local structures.

We solved the linear system of Eq. (3) for $\chi_{\mathrm{Fe} 11}^{\prime}$ (and $\left.\chi_{\mathrm{Fe} 11}^{\prime \prime}\right)$ and the bulk $\mathrm{Fe} \chi_{\mathrm{Fe} 1-10}^{\prime}\left(\right.$ and $\left.\chi_{\mathrm{Fe} 1-10}^{\prime \prime}\right)$ fine structures. Figure 4 shows the Fourier transform (FT) of both signals $\chi_{\mathrm{Fe} 11}^{\prime \prime}$ and $\chi_{\mathrm{Fe} 1-10}^{\prime \prime}$, plus that of the fluorescence EXAFS ( $\left.\chi_{\text {fluo }}^{\prime \prime}\right)$. As expected the Fourier transform of $\chi_{\mathrm{Fe} 1-10}^{\prime \prime}$ and $\chi_{\mathrm{fluo}}^{\prime \prime}$ are very similar, whereas the Fe local structure is rather different at the interfaces. For bcc Fe, the first two nearest-neighbor shells have close distances $\left(N_{\mathrm{Fe}}^{1}=8\right.$ neighbors at $2.48 \AA$ and $N_{\mathrm{Fe}}^{2}=6$ at $\left.2.86 \AA\right)$; therefore, their contributions to the EDAFS signal are mixed in the first peak of the Fourier transform. The nearest shells of $\mathrm{Fe}$ at the interfaces are comprised of $\mathrm{Fe}$ and $\mathrm{Ir}$ atoms. The number of $\mathrm{Fe}$ and Ir nearest neighbors in those shells was estimated from the crystallographic structure, assuming a random mixing, and by taking into account the XAFS-like polarization dependence of the EDAFS signal $\left(N_{\mathrm{Fe}}^{1}=5.2, N_{\mathrm{Fe}}^{2}=3.5, N_{\mathrm{Ir}}^{1}=2.1\right.$, and $N_{\mathrm{Ir}}^{2}=2.5$, respectively). The EXAFS phase and amplitude functions

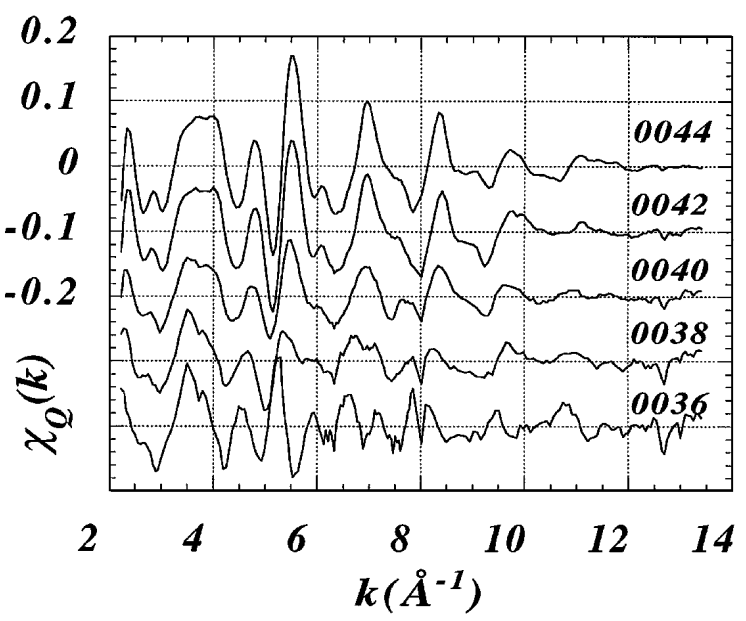

FIG. 3. Normalized EDAFS oscillation spectra $\left[\chi_{\mathbf{Q}}(k)\right]$. The 0038 and 0036 spectra, which have been found to be very sensitive to the Fe-Ir interface, clearly show the existence of different Fe local structure inside the Fe layers. 


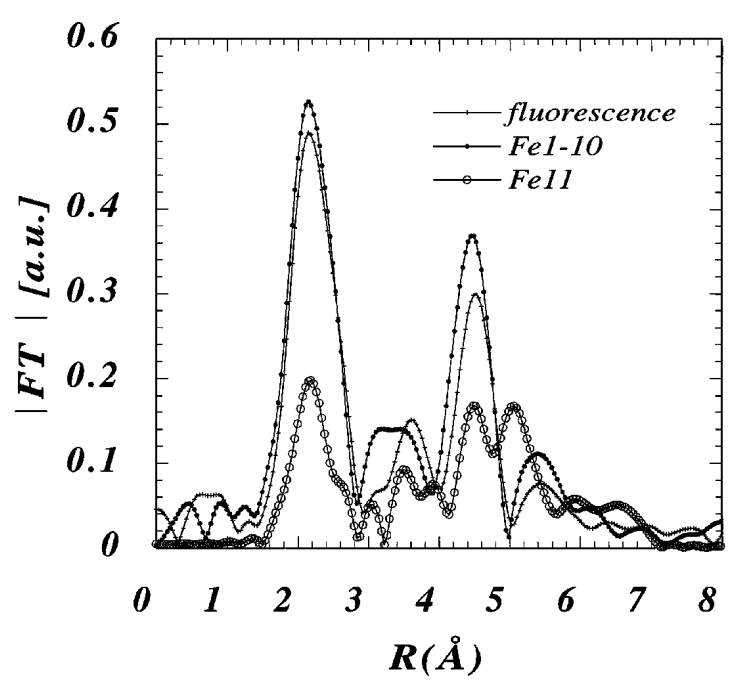

FIG. 4. Fourier transform of the Ir rich $k \chi_{\mathrm{Fe} 11}^{\prime \prime}$ and bulk Fe $k \chi_{\mathrm{Fe} 1-10}^{\prime \prime}$ fine structures. For comparison the Fourier transform of the fluorescence EXAFS $\left(k \chi_{\text {fluo }}^{\prime \prime}\right)$ is also presented. The $k$ range for the FT was 2.5 to $12.5 \AA^{-1}$.

for $\mathrm{Fe}-\mathrm{Fe}$ and $\mathrm{Fe}-\mathrm{Ir}$ were calculated with $f e f f 6.01$ [12] using the refined crystallographic structure. The distances and the mean-square disorder in the bond length $\left(\sigma^{2}\right)$ were refined only. In the end $\mathrm{Fe}-\mathrm{Fe}$ and $\mathrm{Fe}-\mathrm{Ir}$ distances for a given shell were found to be very similar (see Table II). From the back Fourier filtered first nearest-neighbor contribution to $\chi_{\mathrm{Fe} 11}^{\prime \prime}$ the average in-plane first nearest-neighbor distance at the interfaces was found to be 2.87(4) $\AA$, i.e., slightly larger than in the bulk bcc Fe obtained from the back Fourier filtered first nearest-neighbor contribution to $\chi_{\mathrm{Fe} 1-10}^{\prime \prime}$ or $\chi_{\text {fluo }}^{\prime \prime}(2.83 \AA)$. Note that $2.83 \AA$ is a slightly lower value than that of the corresponding distance in $\mathrm{Fe}$ metal $(2.86 \AA)$. The average value of the first nearestneighbor distance was found to be 2.59 (2) $\AA$ instead of 2.47(2) $\AA$ for the bulk "bcc Fe." This result agrees well with the increase of the interplanar distance at the interfaces $(1.43$ to $1.62 \AA=[1.64+1.60] / 2)$ showed by the crystallographic refinements and the value of $2.87 \AA$ for the average in-plane first nearest-neighbor distance. Note that the drastic change of the nearest-neighbor peak of $\chi_{\mathrm{Fe} 11}^{\prime \prime}$ (with respect to the $\chi_{\mathrm{Fe} 1-10}^{\prime \prime}$ one) is to a large extent the result of the increase of the first nearest Fe-Fe (Fe-Ir) distance $(2.59 \AA)$ and the presence of Ir atoms in the nearest shells. The tetragonal distortion at the interface plays a secondary role. Although the filtered signals do show

TABLE II. DAFS extracted Fe nearest-neighbor distances and coordination numbers for the first and second shells.

\begin{tabular}{lcccc}
\hline \hline \multicolumn{1}{c}{ Sites } & $N^{1}$ & $N^{2}$ & $d_{\mathrm{Fe}-X}^{1}(\AA)$ & $d_{\mathrm{Fe}-X}^{2}(\AA)$ \\
\hline Fe1-10 & 8 & 6 & $\mathrm{Fe}-\mathrm{Fe}: 2.47(2)$ & $\mathrm{Fe}-\mathrm{Fe}: 2.83(2)$ \\
$\mathrm{Fe} 11$ & 7.3 & 6 & $\mathrm{Fe}-\mathrm{Fe}: 2.59(2)$ & $\mathrm{Fe}-\mathrm{Fe}: 2.89(4)$ \\
$\mathrm{Fe} 11$ & 7.3 & 6 & $\mathrm{Fe}-\mathrm{Ir}: 2.60(3)$ & $\mathrm{Fe}-\mathrm{Ir}: 2.85(4)$ \\
\hline \hline
\end{tabular}

the presence of Ir atoms in the two nearest-neighbor shells the $\mathrm{Fe}-\mathrm{Fe}$ contribution dominates in the available range of $k\left(2.5 \leq k \leq 12.2 \AA^{-1}\right)$. To obtain more details about Ir shells it would be necessary to measure the EDAFS spectra up to higher $k$ values (at least up to $16 \AA^{-1}$ ) and/or use the Fe $K$-edge DAFS and Ir $L_{\mathrm{III}}$-edge EXAFS data in a multi-refinement procedure.

In conclusion we have used DAFS spectroscopy for studying the different $\mathrm{Fe}$ local structure inside an $\mathrm{Fe} / \mathrm{Ir}(100)$ superlattice. We have shown for the first time that it is possible to probe selectively $\mathrm{Fe}$ atoms at the Ir-Fe interfaces (i.e., $6 \%$ of the total amount of $\mathrm{Fe}$ in the cell) and extract information about the $\mathrm{Fe}$ local environment. Although only $00 \mathrm{l}$ reflections were used, the polarization dependence of DAFS allowed us to obtain the first and second (in the plane of growth) nearest-neighbor distances of $\mathrm{Fe}$ atoms at the Ir-Fe interface. The magnetic properties [13] of $\mathrm{Fe}$ at the interface (hyperfine field distribution, quadrupolar effect) agree well with the existence of different Fe local atomic environments and structures as demonstrated by the DAFS experiments. We have also pointed out the capabilities of a crystallographic-based analysis which allows us to benefit from DAFS spectra and diffraction data for merging the most accurate 3D description of both average and local structures.

We are grateful to Dr. D. Aberdam, Dr. A. Bienenstock, and Dr. D. Raoux for helpful discussions and encouragement.

*Corresponding author.

Electronic address: renevier@polycnrs-gre.fr

[1] (a) Y. Cauchois and C. Bonnelle, C. R. Acad. Sci. 242, 1596 (1956); (b) I. Arcon et al., J. Phys. C 9, 1105 (1987).

[2] H. J. Stragier et al., Phys. Rev. Lett. 69, 3064 (1992).

[3] H.J. Stragier, Ph.D. thesis, University of Washington, 1993.

[4] H. Renevier et al., Physica (Amsterdam) 208B\&209B, 215 (1995).

[5] I. J. Pickering et al., J. Am. Chem. Soc. 115, 6302 (1993).

[6] J. Vacinova et al., J. Synchrotron Radiat. 2, 236 (1995); J. L. Hodeau et al., Nucl. Instrum. Methods Phys. Res., Sect. B 97, 115 (1995).

[7] For a complete review, see S. Andrieu et al., Phys. Rev. B 52, 9938 (1995).

[8] S. Andrieu et al., Europhys. Lett. 26, 189 (1994).

[9] R. Frahm et al., Rev. Sci. Instrum. 66, 1677 (1994).

[10] M. Roth et al., Rev. Sci. Instrum. 63, 1043 (1992); J. P. Simon et al., Rev. Sci. Instrum. 63, 1051 (1992).

[11] D. T. Cromer, J. Appl. Crystallogr. 16, 437 (1983).

[12] J. Mustre de Leon et al., Phys. Rev. B 44, 4146 (1991); S. I. Zabinsky et al., Phys. Rev. B 52, 2995 (1995).

[13] Ph. Bauer et al., in Proceedings of the E-MRS Spring Meeting Conference, Strasbourg, 1996 [J. Magn. Magn. Mater. (to be published)]. 